\title{
Métodos de análise conceitual na enfermagem: uma reflexão teórica
}

\author{
Conceptual analysis methods in nursing: a theoretical reflection \\ Métodos de análisis conceptual en enfermería: una reflexión teórica
}

Valéria Silvana Faganello Madureira ${ }^{1,2}$ (]) Denise Maria Guerreiro Vieira da Silva ${ }^{2,3}$ (1) Mercedes Trentini ${ }^{2}$ (1) Sabrina da Silva de Souza ${ }^{4,5}$ []

\section{Universidade Federal da Fronteira Sul.} Chapecó, SC, Brasil.

2. Universidade Federal de Santa Catarina, Programa de Pós-graduação em Enfermagem. Florianópolis, SC, Brasil.

3. Universidade do Estado do Amazonas, Programa de Pós-Graduação em Enfermagem em Saúde Pública. Manaus, AM, Brasil.

4. Secretaria Municipal de Saúde de São José. São José, SC, Brasil.

5. Universidade Federal de Santa Catarina, Hospital Universitário, Programa de Pós-Graduação em Gestão do Cuidado. Florianópolis, SC, Brasil.
Autor Correspondente:

Valéria Silvana Faganello Madureira E-mail: valeriamadureira2005@hotmail.com

Recebido em 25/05/2020.

Aprovado em 15/10/2020.

DOI:https://doi.org/10.1590/2177-9465-EAN-2020-0186

\section{Resumo}

Objetivo: refletir sobre diferentes métodos de análise conceitual utilizados na enfermagem. Método: Identificaram-se os métodos com os critérios: nível de detalhamento, distinções com outras propostas e uso em estudos da enfermagem. Elaborou-se um quadro com oito métodos de análise de conceito, sintetizando-os, analisando-os e destacando seus aspectos centrais. Resultados: Identificaram-se duas 'linhas' de metodologias de análise. A primeira delas é estruturada, com passos objetivos e pouco flexíveis. A segunda, por sua vez, flexibiliza o percurso de pesquisa, que é construído/modificado no processo. Conclusão e implicação para a prática: A análise conceitual mostra-se útil para avaliar como um conceito vem sendo usado na enfermagem, quais modificações se processam com seu uso, ao longo do tempo, a que situações se aplica. A escolha por um método depende do nível de desenvolvimento do conceito, reforçando a proposição de que há uma gradação, ou seja, existem conceitos que já são amplamente utilizados pela enfermagem e outros que estão surgindo nas falas e textos da disciplina.

Palavras-chave: Formação de conceito. Enfermagem. Pesquisa em enfermagem. Teoria de Enfermagem.

\section{Abstract}

Objective: to reflect on different conceptual analysis methods used in nursing. Method: The methods were identified with the criteria: level of detail, distinctions with other proposals and use in nursing studies. It was elaborated a chart with eight methods of concept analysis, in synthesizing them, analyzing them and highlighting their central aspects. Results: Two 'lines' of analysis methodologies were identified. The first one is structured, with objective and not very flexible steps. The second, in turn, makes the research path more flexible, which is built/modified in the process. Conclusion and implication for practice: Conceptual analysis is useful to assess how a concept has been used in nursing, what changes are processed with its use over time, to which situations it applies. The choice for a method depends on the level of development of the concept, reinforcing the proposition that there is a gradation, that is, there are concepts already being widely used by nursing and others that are appearing in the discipline's speeches and texts.

Keywords: Concept formation. Nursing. Nursing research. Nursing Theory.

\section{RESUMEN}

Objetivo: reflexionar sobre los diferentes métodos de análisis conceptual utilizados en enfermería. Método: Se han identificado los métodos con los criterios: nivel de detalle, distinciones con otras propuestas y uso en estudios de enfermería. Se elaboró una tabla con ocho métodos de análisis de conceptos, sintetizándolos, analizándolos y destacando sus aspectos centrales. Resultados: Se han identificado dos "líneas" de metodologías de análisis. La primera está estructurada, con pasos objetivos y poco flexibles. La segunda, a su vez, flexibiliza el recorrido de la investigación, que es construido / modificado en el proceso. Conclusión e implicación para la práctica: El análisis conceptual es útil para evaluar cómo se ha utilizado un concepto en enfermería, qué cambios se producen con su uso a lo largo del tiempo, a qué situaciones se aplica. La elección de un método depende del nivel de desarrollo del concepto, reforzando la proposición de que existe una gradación, es decir, que hay conceptos que ya son ampliamente utilizados por la enfermería y otros que están apareciendo en las conversaciones y textos de la disciplina.

Palabras clave: formación de conceptos. Enfermería. Investigación en enfermería. Teoría de enfermería. 


\section{INTRODUÇÃO}

A enfermagem é ciência jovem e vem ampliando sua base de conhecimentos científicos para fundamentar a pesquisa, o ensino e a prática da profissão. Nesse sentido, o desenvolvimento de teorias focaliza fenômenos importantes, propondo-se a embasar o cuidado de enfermagem em diferentes cenários e situações. Inicialmente, a base conceitual da teoria e da pesquisa em enfermagem utilizou conceitos de outras disciplinas, o que tornou necessário verificar sua adequação, pois muitas definições podem ter sido alteradas durante o seu processo de aplicação. ${ }^{1}$ Conceitos embasam o pensamento teórico na Enfermagem ${ }^{2} \mathrm{e}$ é necessário que possam ser claramente compreendidos para que ampliem o conhecimento da disciplina, sejam traduzidos na pesquisa e na prática profissional. ${ }^{3}$

Os conceitos delimitam e guiam a disciplina, formando unidades que vinculam teoria, pesquisa e prática em enfermagem. ${ }^{4}$ $\mathrm{Na}$ enfermagem, conceitos comportamentais são de maior interesse para pesquisadores e podem ser: ${ }^{5}$ cotidianos, usados na linguagem comum e descritos em dicionários; e científicos, desenvolvidos por pesquisadores cujas definições são restritas. Entretanto, a dinamicidade da linguagem absorve um conceito científico no cotidiano e vice-versa.,6 Daí a importância de analisar conceitos que surjam na literatura para estabelecer a utilidade pragmática destes. $^{5}$

Dessa forma, análise de conceito ou conceitual se refere ao processo empregado para elucidar significados, a fim de que possam ser compreendidos de maneira similar por aqueles que utilizam o conceito em um mesmo contexto, recorte espaço-temporal e situação. É parte do processo de formação de conceito e envolve formulação e esclarecimento de um "construto mental, sistematizando informações relevantes de maneira a permitir sua avaliação e aprimoramento" para avanço da teoria e para orientar a prática. ${ }^{4}$

A busca por um método para proceder à análise de conceitos em um estudo em desenvolvimento pelas autoras revelou a existência de inúmeros deles, propostos por enfermeiros ou para uso na enfermagem. A leitura atenta indicou que, embora todos tenham propostas similares, com distinções que possam parecer inicialmente sutis, vão sendo desveladas no decorrer do processo. Já o aprofundamento da compreensão mostrou que tais diferenças podem ser relevantes na condução da análise conceitual. Considerando a dificuldade encontrada neste percurso, estudaram-se detalhadamente diferentes métodos de análise conceitual utilizados na enfermagem, colocando em relevo os aspectos que caracterizam cada um, de forma a facilitar a escolha por cada um deles. Dessa intenção, nasceu o presente artigo, com o objetivo de refletir sobre diferentes métodos de análise conceitual utilizados na enfermagem.

\section{Análise Conceitual e os Caminhos Percorridos nesta Reflexão}

Há diferentes definições para a palavra 'conceito': a) palavra ou frase que resume uma ideia, observação ou experiência (fenômeno), provendo uma imagem mental que facilita a compreensão do fenômeno e a comunicação sobre ele; ${ }^{7}$ b) abstração expressa de forma discursiva ou não que, pela socialização e interação pública, associa-se a atributos que o definem; ${ }^{8} \mathrm{c}$ ) construção mental que atribui significado a estímulos ambientais na tentativa de ordená-los;9,10 d) rótulo usado para descrever um fenômeno ou grupo de fenômenos; ${ }^{3}$ e) como os tijolos básicos (the basic building blocks) de uma teoria ${ }^{9-13} \mathrm{e}$ f) fios que compõem uma tapeçaria. ${ }^{14} A$ diversidade de definições demonstra a polissemia do termo e aponta para a variedade de métodos utilizados para a análise. Todas as definições são pertinentes ao domínio da enfermagem, a depender do método que se utilize para desenvolver a análise conceitual.

Reflexões sobre análise de conceito já foram publicadas no Brasil. ${ }^{15-18}$ Entretanto, os conceitos mudam, bem como os referenciais e métodos de análise conceitual o que, aliado às lacunas existentes na literatura consultada, dificulta a escolha de um método. Com relação à análise conceitual, há os que a defendem como essencial no avanço do conhecimento da enfermagem ou como importante ponto inicial de trabalhos acadêmicos. ${ }^{19}$ As críticas apresentam diferentes argumentos: acreditam que essa prática tenha sido adequada nos anos 1980, no bojo do movimento científico para a produção de teorias de enfermagem, mas já não o é atualmente; ${ }^{20}$ defendem a ideia de que teorias são criadas a partir de questões teóricas e/ou práticas para posteriormente serem testadas e validadas por meio de pesquisa; afirmam que há falta de vínculo dos métodos com a filosofia da ciência. ${ }^{20,21}$ Este último argumento se fundamenta na ideia de que teorias resultam do esforço criativo empreendido para propor soluções para problemas de origem prática e/ou científica e não da análise de conceitos. ${ }^{21}$ Há ainda quem considere que a análise de conceitos precisa manter vínculo claro com a resolução de problemas da disciplina. ${ }^{22}$

Neste cenário, esta reflexão foi empreendida ao considerar tais críticas e a relevância dos conceitos no avanço do conhecimento em enfermagem. Para tanto, foram lidos livros e artigos que tratam de análise conceitual e de metodologias utilizadas para desenvolvê-la, o que possibilitou identificar métodos frequentemente usados na enfermagem..$^{9,10,23,24} \mathrm{Na}$ leitura dos originais desses autores e de críticas à análise conceitual, outros autores foram identificados. $3,5,6,11,12,25-29$

Assim, foram observados níveis diferentes de detalhamento nos métodos propostos, distinções entre eles e diferentes intensidades de uso nos estudos. Esses aspectos, aliados ao emprego na enfermagem e à clareza da metodologia, nortearam a decisão sobre aqueles a serem incluídos nesta reflexão. Foram selecionados oito métodos de autores internacionais (Inglaterra, Finlândia e Estados Unidos - EUA), dentre os quais cinco atuam nos EUA. Os métodos apresentados foram publicados a partir de 1963 com atualizações até 2019, com maior concentração de produções entre 1980 e 2000. Dois estudos não foram realizados na enfermagem: um deles foi proposto na década de 1960 na Inglaterra ${ }^{29}$ e o outro teve como base publicações da enfermagem sobre análise conceitual. ${ }^{26-28}$ 
Para apresentar o resultado, elaborou-se um quadro comparativo com oito métodos de análise de conceito, sintetizando-os, analisando-os e destacando seus aspectos centrais. Entretanto, é importante ressaltar que, além desses, há outros métodos que não foram incluídos por: terem semelhanças entre si; não argumentarem consistentemente sobre suas bases filosóficas e ou por não estarem suficientemente apresentados.

\section{MÉTODOS DE ANÁLISE CONCEITUAL}

Os métodos analisados seguem sintetizados no Quadro 1.

\section{REFLEXÕES}

Foi possível observar duas 'linhas' de metodologias de análise de conceito dentre as analisadas. Uma delas, mais estruturada, tem passos mais objetivos e menos flexíveis, embora se sobreponham e está representada por: John Wilson; ${ }^{29}$ Lorraine Walker e Kay Avant; 9,10 Rodgers ${ }^{22}$ e Anita Nuopponen. ${ }^{26-28}$ É aplicável quando se deseja adotar um percurso linear, quando se inicia em análise conceitual ou, ainda, quando o pesquisador desenvolve estudos e pesquisas estruturados. A segunda 'linha', mais flexível, considera que o percurso é construído/modificado no processo de pesquisa e inclui: Morse (2000): ${ }^{1,5}$ Penrod e Hupcey (2005); ;,14 Donna Schwartz-Barcott e Hesook S. Kim (1986, 2000); ;3 Meleis (1997). ${ }^{3}$

Criado no campo da educação, o método proposto por Wilson ${ }^{29}$ defende que palavras servem a propósitos humanos e, para isso, o significado deve ser adequado à situação e ao contexto. É a principal referência para métodos na enfermagem e inspirou propostas de análise conceitual a exemplo de Walker e Avant e Schwartz-Barcott e Kim. Há quem afirme que o método de Rodgers é wilsoniano ${ }^{1,13,30}$ e há quem afirme que não. ${ }^{4}$ O método por ele proposto recebeu várias críticas que se estendem àqueles que derivaram dele e, nesse sentido, diz-se que o livro didático de Wilson 29 "foi levado mais a sério pelos acadêmicos de enfermagem do que merecia, mesmo a um ponto de simplificação excessivamente enganosa". 4:183 Entretanto, Wilson considera que as etapas 'contexto social, resultados práticos ou ansiedade subjacente' não são necessários em todas as análises.

Sobre Walker e Avant, por exemplo, existem críticas relacionadas ao fato de que o método simplifica a complexidade do desenvolvimento conceitual, produzindo frequentemente resultados triviais e insignificantes..$^{30}$ Outras críticas apontam a

QUADRO 1: Síntese dos métodos de análise conceitual selecionados para o estudo

\begin{tabular}{|c|c|}
\hline Autor(es) & Descrição \\
\hline $\begin{array}{l}\text { John Wilson } \\
1963\end{array}$ & $\begin{array}{l}\text { Proposta para ajudar estudantes de ensino médio a compreender o significado das } \\
\text { palavras e melhorar a comunicação, tornando o pensamento mais estruturado e } \\
\text { objetivo. Inspiração teórica não descrita, mas, depreende-se influência do paradigma } \\
\text { positivista. Análise com } 11 \text { passos: } 1 \text {. Isolar perguntas sobre conceitos, diferenciando-os } \\
\text { de fatos e de valores. } 2 \text {. Buscar respostas certas. 3. Casos-modelo. 4. Caso contrário, } \\
\text { Contraexemplo. 5. Casos afins ou relacionados. 6. Casos limítrofes. 7. Casos inventados. } \\
\text { 8. Contexto social. 9. Ansiedade subjacente. 10. Resultados práticos. 11. Consequências } \\
\text { na linguagem. }\end{array}$ \\
\hline $\begin{array}{l}\text { Lorraine O. Walker } \\
\text { Kay C. Avant } \\
\text { 1983, } 2019 \\
\text { Estados Unidos } \\
\text { Adaptação de Wilson para a } \\
\text { Enfermagem. }\end{array}$ & $\begin{array}{l}\text { Considerado como estratégia para examinar atributos centrais de um conceito, } \\
\text { possibilitando a identificação clara do fenômeno a que se refere. Análise com oito } \\
\text { passos: } 1 \text {. Selecionar um conceito. } 2 \text {. Definir objetivos da análise. 3. Identificar usos } \\
\text { possíveis do conceito. } 4 \text {. Determinar atributos definidores identificados. } 5 \text {. Construir } \\
\text { ao menos um caso- modelo. } 6 \text {. Construir casos adicionais, exemplificando o que o } \\
\text { conceito NÃO É (limítrofe; relacionado; contrário; inventado; ilegítimo). } 7 \text {. Identificar } \\
\text { antecedentes e consequentes. 8. Definir referências empíricas dos atributos definidores. }\end{array}$ \\
\hline $\begin{array}{l}\text { Método evolucionário de análise } \\
\text { de conceito }\end{array}$ & $\begin{array}{l}\text { Propõe-se a elucidar conceitos, avaliando sua robustez, limitações e introduzindo } \\
\text { variações que ampliem sua contribuição para alcançar objetivos intelectuais. Na análise, } \\
\text { são essenciais: significância, uso e aplicação, que progridem em ciclo contínuo através } \\
\text { do tempo e de acordo com o contexto, o que caracteriza a concepção evolucionária. } \\
\text { Baseou-se no pensamento de H.H. Price, Richard Rorty, Stephen Toulmin e nos escritos } \\
\text { tardios de Ludwig Wittgenstein. Análise em sete etapas: } 1 \text {. Identificar e nomear o } \\
\text { conceito de interesse. } 2 \text {. Identificar termos substitutos e usos relevantes do conceito. } \\
\text { 3. Identificar e selecionar uma amostra para a coleta de dados, na enfermagem ou } \\
\text { incluindo outras disciplinas. } 4 \text {. Identificar os atributos do conceito. 5. Identificar } \\
\text { referentes, antecedentes e consequentes. } 6 \text {. Identificar conceitos relacionados. } \\
\text { 7. Identificar um caso modelo (exemplo real). }\end{array}$ \\
\hline
\end{tabular}

Fonte: Banco de dados autoras (2019) 
QUADRO 1: Continuação...

\begin{tabular}{|c|c|}
\hline Autor(es) & Descrição \\
\hline $\begin{array}{l}\text { Donna Schwartz-Barcott } \\
\text { Hesook S. Kim } \\
\text { 1986, } 2000 \\
\text { Estados Unidos }\end{array}$ & $\begin{array}{l}\text { Visa a refinar conceitos para desenvolvimento de teorias. Híbrido porque interliga } \\
\text { análise teórica e observação empírica. Indica inspiração em três áreas de conhecimento: } \\
\text { filosofia da ciência, sociologia da construção de teorias e observação participante ou } \\
\text { pesquisa de campo. Análise com três passos: } 1 \text {. Fase Teórica: seleção do conceito, busca } \\
\text { na literatura, busca de significados e instrumentos de medida do conceito, para escolher } \\
\text { definição a ser usada na fase de campo. } 2 \text {. Trabalho de Campo: seleção de um campo, } \\
\text { negociação da entrada no campo, seleção de casos, coleta e análise de dados. 3. Fase } \\
\text { Analítica: analisar e ponderar os dados, redigir os achados. }\end{array}$ \\
\hline $\begin{array}{l}\text { Anita Nuopponen } \\
1994,2016 \\
\text { Finlândia } \\
\text { Análise sistemática de conceito } \\
\text { Utilizou estudos desenvolvidos } \\
\text { na enfermagem para propor seu } \\
\text { método }\end{array}$ & $\begin{array}{l}\text { Esclarece e descreve conceitos pertencentes a um todo, suas características e as relações } \\
\text { que mantêm dentro dos sistemas de conceitos. Parte da discussão de métodos de } \\
\text { análise de conceito usados em estudos de negócios (business studies) e na ciência da } \\
\text { enfermagem. Análise em seis passos: 1) Objetivo, delimitação do domínio e do número } \\
\text { de conceitos a serem tratados. 2) Aquisição de conhecimento de domínio e criação } \\
\text { de uma ideia geral do campo. 3) Compilação do material. 4) Elaboração de sistema } \\
\text { conceitual preliminar e/ou marco para análise. 5) Análise sistemática do material, } \\
\text { envolvendo seis etapas. 6) Análise e conclusões adicionais de acordo com o objetivo da } \\
\text { análise do conceito }\end{array}$ \\
\hline $\begin{array}{l}\text { Janice M. Morse } \\
2000 \\
\text { Estados Unidos }\end{array}$ & $\begin{array}{l}\text { Processo de investigação que tem na literatura a fonte de dados para explorar a utilidade } \\
\text { pragmática do conceito. Os resultados promovem o avanço do conhecimento para } \\
\text { além do que é conhecido. Inspirações teóricas não claras. Baseou-se em publicações } \\
\text { anteriores de sua autoria ou coautoria. Destaca as 'regras de relação' propostas por } \\
\text { Bolton (1977) para identificar o significado e a maturidade do conceito. Adota princípios } \\
\text { orientadores para a análise: 1) estabelecer propósito claro da investigação, com questão } \\
\text { preliminar para nortear a busca de literatura. Delimitar o conceito a ser explorado; } \\
\text { identificar todos os termos usados, compondo lista de termos de busca e identificando } \\
\text { conceitos relacionados e contrários, sem incluir significados alternativos. 2) assegurar } \\
\text { a validade, buscando na literatura as bases relevantes de dados e desenvolver } \\
\text { estratégias para acessar o sistema de registro de dados. 3) identificar questões analíticas } \\
\text { significantes, tornando-se familiarizado com a literatura pela leitura de tudo o que for } \\
\text { encontrado para refinar a questão de pesquisa, o que determinará a organização dos } \\
\text { dados para a comparação e subsidiará a identificação de questões analíticas. 4) Sintetizar } \\
\text { resultados, preparando o 'cenário' para visualizar o todo e compilar os resultados. }\end{array}$ \\
\hline $\begin{array}{l}\text { Afaf I. Meleis } \\
1997 \\
\text { Estados Unidos } \\
\text { Abordagem Integrada para o } \\
\text { Desenvolvimento de Conceitos }\end{array}$ & $\begin{array}{l}\text { Analisa conceitos bem definidos na literatura da enfermagem para elevar o nível de } \\
\text { compreensão, visando ao desenvolvimento de teorias. A análise de conceito guia o que } \\
\text { vemos e ordena situações e eventos. Reconhece o pioneirismo na análise de conceito } \\
\text { de Wilson, Walker e Avant e Rodgers e Knafl. Análise com seis estágios: 1) Levando } \\
\text { (taking in): dimensionar uma situação que chama a atenção e dar atenção a ela. } \\
\text { 2) Descrevendo o fenômeno. 3) Rotulando (labeling). 4) Desenvolvimento do conceito. } \\
\text { 5) Desenvolvimento de declaração. 6) Suposições explicativas. }\end{array}$ \\
\hline $\begin{array}{l}\text { Janice Penrod } \\
\text { Judith E. Hupcey } \\
2005 \\
\text { Estados Unidos }\end{array}$ & $\begin{array}{l}\text { O foco é integrar o que é conhecido sobre o conceito, atuando para ampliar a } \\
\text { compreensão de um fenômeno de interesse. Identifica lacunas e inconsistências } \\
\text { na compreensão de um conceito. Proposta guiada por quatro princípios filosóficos } \\
\text { propostos por Morse (1995) e Hupcey et al. (1996): epistemológico; pragmático; } \\
\text { linguístico; lógico. Propõe análise em três etapas, ressaltando a necessidade de } \\
\text { utilizar os princípios filosóficos em cada etapa metodológica: 1) Seleção da literatura. } \\
\text { 2) Amostragem dirigida pelo conceito. 3) Análise intra e interdisciplinar. O resultado } \\
\text { será uma definição teórica que integre a compreensão científica do fenômeno e } \\
\text { recrie a tapeçaria teórica do conceito. Lacunas e inconsistências científicas podem ser } \\
\text { identificadas, aumentando o poder da análise para o avanço da ciência da enfermagem. }\end{array}$ \\
\hline
\end{tabular}

Fonte: Banco de dados autoras (2019) 
falta de delineamento filosófico, ontológico, de contextualização na análise, além de ser considerado reducionista e rígido. Para Walker e Avant, o método é ímpar para aprendizagem de estudantes e é necessário que a análise amplie a aplicação do conceito na prática e na pesquisa para que possa ser publicada. ${ }^{10}$ Com isso, estendem a responsabilidade pela ampliação da base de conhecimentos da Enfermagem às revistas e jornais científicos. No método por elas proposto, a análise de conceito tem valor na medida em que contribui para pesquisas e desenvolvimento de teorias. Além disso, a análise deve ter repercussão na prática de enfermagem. Porém, apesar das críticas que tem recebido, é um dos métodos mais utilizados pela enfermagem.

Beth Rodgers ${ }^{8,24}$ propôs o método evolucionário e rejeita semelhanças com Wilson e Walker e Avant devido à explicitação filosófica e epistemológica de sua proposta, embora reconheça possíveis similaridades entre os processos. Rodgers considera inapropriada a identificação ou construção de outros casos (limítrofe, contrário, inventado e ilegítimo), tal como o fazem outras propostas. ${ }^{9,10,23} \mathrm{O}$ caso-modelo que recomenda construir ao final da análise deve ser verdadeiro. A possibilidade de utilizar entrevistas para captação de dados é também uma diferença e favorece a contextualização do conceito. Ressalta a característica mutável dos conceitos no tempo e com o contexto; por isso devem ser interpretados em um dado contexto, o que parece limitá-los a ele, o que pode ser considerado uma fraqueza do método, ${ }^{4}$ embora seja coerente com os princípios de pesquisa qualitativa que utiliza, cuja generalização de resultados não é central.

No desenvolvimento de um conceito, Rodgers considera significância, uso e aplicação, ${ }^{24}$ que progridem em um ciclo contínuo através do tempo. Significância refere-se à habilidade de auxiliar na resolução de problemas e caracterizar adequadamente os fenômenos. Um conceito frequentemente usado é considerado significativo e seu uso se refere aos atributos que ajudam a organizar a existência humana. A significância impacta a frequência e a extensão do uso, na forma como o conceito é aplicado, nas situações apropriadas para sua aplicação e no uso na linguagem ou em outra forma de apresentação. ${ }^{24}$

O trabalho de Schwartz-Barcott e $\mathrm{Kim}^{23}$ é considerado derivação de Wilson que avança ao propor a articulação entre as fases teórica, de campo e analítica, o que o diferencia da proposta de Walker e Avant e Rodgers. No modelo de fases sobrepostas, o conceito deve estar vinculado a um cenário de cuidado e sempre emergir da prática clínica. A coleta de dados em campo é essencial para elucidar ou desenvolver um conceito, o que é feito seguindo princípios da pesquisa qualitativa, com a criatividade e a inovação do pesquisador, assumindo centralidade na análise. Entretanto, a condução da análise de conceito não fica clara. Depreende-se que o conceito escolhido da prática e previamente definido com base na literatura será modificado, elucidado e refinado pela análise dos dados oriundos da fase de campo. Porém, a vinculação com Wilson surge quando a aplicação da tipologia de casos proposta por ele é indicada para situações em que as características essenciais e os indicadores do conceito não estejam claros. Não há esclarecimento sobre como tais características e indicadores são encontrados.

$\mathrm{Na}$ proposta de análise sistemática de Nuopponen, ${ }^{26,28} \mathrm{a}$ ideia de fundo é que "nenhum conceito é uma ilha" e que toda análise envolve outros conceitos, cujas interrelações devem ser esclarecidas e apresentadas em diagrama gráfico. A análise é uma atividade na qual conceitos pertencentes a um todo, suas características e as relações que mantêm dentro dos sistemas de conceitos são esclarecidos e descritos. É aplicável a muitos propósitos, combinando elementos de análise terminológica com elementos de outros métodos de análise de conceito mais orientados para a pesquisa. Porém, somente a análise do conceito central é levada em conta e a orientação para o trabalho de terminologia é um dos propósitos para os quais a análise sistemática pode ser usada. Indica diagramas como ferramentas que reúnem tipos de conceitos e relações conceituais através de tipologias para formar sistemas conceituais mistos. A base desse conjunto de ferramentas é a construção de um ou mais mapa(s) ou sistema(s) conceitual(ais), a partir da compilação do conhecimento e da estruturação do campo. Tais ferramentas incluem: Modelo de satélite (conhecimento gráfico de mapas mentais e apresentação de conceitos) e Modelo de relação conceitual (modelos básicos e estruturais, de originação, desenvolvimento, atividade, transmissão, causalidade e dependência). Não foram encontrados estudos desenvolvidos na enfermagem com utilização da análise sistemática.

No método 'Utilidade Pragmática de Conceitos' de Morse, ${ }^{5}$ os atributos, as características e os limites de um conceito são identificados na literatura, a qual deve ser buscada em um recorte amplo de tempo, incluindo o que já tenha sido publicado sobre o conceito e com leitura completa de todo o material. A leitura completa de toda a literatura encontrada é central para avaliar a maturidade de um conceito. É considerado maduro um conceito que tenha, a seu respeito, literatura adequada disponível em número e qualidade. O resultado leva o conhecimento para além do que é atualmente conhecido sobre o conceito e "revela novos insights, coloca questões significativas e fornece orientações sobre qual nível de investigação e quais métodos devem ser usados na próxima fase". $5: 334$ Segundo Morse, ${ }^{5}$ os princípios orientadores que propõe visam a superar a simplificação do procedimento comum a outros métodos, aos quais tecem-se muitas críticas e a valorizar o trabalho intelectual envolvido na análise. Os princípios, amplos e genéricos exigem habilidades em pesquisa qualitativa para definição progressiva da questão de pesquisa, de critérios de seleção e das questões analíticas que nortearão a análise. O que é necessário para que os resultados deem suporte ao pesquisador para redefinir o conceito. É um método complexo que demanda longo tempo para ser desenvolvido e que parece mais adequado aos pesquisadores experientes que se dediquem a estudos conceituais.

Para Meleis, ${ }^{3} a$ análise de conceito guia o que vemos e ajuda a ordenar situações e eventos. Na Abordagem Integrada para o Desenvolvimento de Conceitos que propõe, as estratégias permitem que o pesquisador desenvolva a análise de acordo 
com a situação do conceito de interesse na enfermagem. O processo de análise, que visa ao desenvolvimento de conceitos, baseia-se nas limitações dos demais métodos em capturar o contexto, o que o aproxima de Rodgers e Schwartz-Barcott e Kim. ${ }^{9,23,24}$ Ressalta que outros métodos também falham em captar os vieses existentes na estrutura social, tais como sexismo, política e racismo. Estes casos não são considerados sob a perspectiva da prática clínica ou da experiência, mas sob a ótica de receitas que reduzem o processo à condição de ingredientes, passos e fases, sem explorar o pensamento crítico, conscientização e clarificação de valores. Ressaltam, ainda, a existência de gradação no tipo de análise, a depender do nível de maturidade do conceito: a) exploração de conceito, para conceitos novos ou usados acriticamente; 2) elucidação de conceito, quando não há concordância clara, compartilhada e consciente de significados e c) para aprofundar conceitos bem definidos na literatura. Aproxima-se de Morse ao abordar o nível de maturidade de um conceito, porém não limita sua proposta de análise àqueles conceitos já maduros.

$\mathrm{Na}$ análise baseada em princípios de Penrod e Hupcey, ${ }^{6,14}$ conceitos são abstrações da realidade baseadas em dados empíricos, que não assumem forma concreta e cuja formação é resultado do ser-estar no mundo com os outros e não apenas resultados de esforços científicos. ${ }^{6} \mathrm{~A}$ 'análise de conceito' é ferramenta para ampliar a compreensão de um fenômeno de interesse, enquanto o 'aperfeiçoamento de conceito' visa a refinar um conceito, ampliando sua compreensão científica. ${ }^{6}$ Conceitos são entrelaçados para formar uma teoria, tal como os fios de uma tapeçaria. Daí a importância do contexto teórico na análise conceitual, que identificará as vertentes teóricas definidoras do conceito, para que seja possível atar e reatar os nós, formando uma tapeçaria teórica mais forte e coerente. A teoria (tapeçaria) é fortalecida à medida que cada fio (conceito) é esclarecido e desenvolvido. ${ }^{6} \mathrm{Na}$ proposta, a análise crítica da literatura científica produz evidências que revelam o estado da ciência sobre o conceito, a melhor estimativa dos estudiosos a respeito da 'verdade provável' em torno dele, resultando em definição que integre a compreensão científica do fenômeno e recrie a tapeçaria teórica do conceito. O método revela o que é conhecido sobre o conceito em um determinando ponto no tempo. A identificação de lacunas e inconsistências na conceitualização científica aumenta o poder da análise conceitual para o avanço da enfermagem como ciência. ${ }^{14}$ Portanto, esse método delineia o panorama do que já é conhecido sobre o conceito com base na literatura multidisciplinar disponível, reconhecendo o estado da arte a respeito dele e possibilitando que sua utilização em novos estudos amplie o estado da ciência sobre ele. Ao trazer como resultado o que já existe sobre um conceito, diferencia-se de Morse, cujo método propõe ampliar o que se conhece atualmente sobre o conceito

\section{CONSIDERAÇÕES FINAIS}

Esta reflexão apresentou diferentes métodos de análise conceitual usados na enfermagem, sintetizando-os, analisando-os, destacando seus aspectos centrais e comparando-os entre si. Mesmo com a intenção inicial de indicar métodos que melhor atendessem à enfermagem, chega-se ao final com a compreensão de que a escolha depende do nível de desenvolvimento do conceito, reforçando a proposição de que há uma gradação, ou seja, conceitos que já são amplamente utilizados pela enfermagem e aqueles que estão surgindo nas falas e escritos da disciplina. Além disso, a perspectiva filosófica e conceitual do pesquisador influencia a escolha do método.

Ao estudar análise conceitual fica-se com a impressão de que pode ser atividade muito complexa e com etapas amplas, com subsequentes análises que sugerem um ciclo inesgotável em que se procura aprofundar cada vez mais a clareza de um conceito. É, sem dúvida, importante esclarecer conceitos, desde que esta empreitada intelectual não seja um fim em si mesma e tenha intencionalidade clara: desenvolver teorias de enfermagem, ampliar a compreensão de um conceito, contextualizar sua aplicação em uma dada realidade, atualizá-lo a partir das modificações que sofreu ao ser aplicado.

Enfim, entende-se que a análise conceitual é útil para avaliar a forma como um conceito vem sendo utilizado na enfermagem, se houve modificações em seu uso ao longo do tempo, a que situações se aplica. Porém, o olhar do pesquisador deve se estender para além da análise, justamente para que não se perca em semânticas e volte-se, de algum modo, para a prática e para a pesquisa em enfermagem. A elucidação de um conceito pode conduzir a formas de avaliação, de medida e, por extensão, a formas de cuidar.

\section{FINANCIAMENTO}

O presente trabalho foi realizado com apoio da Coordenação de Aperfeiçoamento de Pessoal de Nível Superior - Brasil (CAPES) - Código de Financiamento 001. Bolsa do Programa Nacional de Pós-Doutorado (PNPD), concedida a Valéria Silvana Faganello Madureira. Bolsa de Produtividade em Pesquisa do CNPq, concedida a Denise Maria Guerreiro Vieira da Silva, Processo no 88887.358300/2019-00

\section{CONTRIBUIÇÕES DOS AUTORES}

Desenho do estudo. Valéria Silvana Faganello Madureira. Denise Maria Guerreiro Vieira da Silva.

Levantamento das fontes bibliográficas. Valéria Silvana Faganello Madureira. Denise Maria Guerreiro Vieira da Silva

Análise de informaçòes. Valéria Silvana Faganello Madureira Denise Maria Guerreiro Vieira da Silva

Interpretação dos resultados. Valéria Silvana Faganello Madureira. Denise Maria Guerreiro Vieira da Silva. Mercedes Trentini. Sabrina da Silva de Souza

Redação e revisão crítica do manuscrito. Valéria Silvana Faganello Madureira. Denise Maria Guerreiro Vieira da Silva. Mercedes Trentini. Sabrina da Silva de Souza 
Aprovação da versão final do artigo. Valéria Silvana Faganello Madureira. Denise Maria Guerreiro Vieira da Silva. Mercedes Trentini. Sabrina da Silva de Souza

Responsabilidade por todos os aspectos do conteúdo e a integridade do artigo publicado. Valéria Silvana Faganello Madureira. Denise Maria Guerreiro Vieira da Silva. Mercedes Trentini. Sabrina da Silva de Souza

\section{EDITOR ASSOCIADO:}

\author{
Rafael Celestino da Silva
}

\section{REFERÊNCIAS}

1. Morse JM, Hupcey JE, Mitcham C, Lenz ER. Concept analysis in nursing research: A critical appraisal. Sch Inq Nurs Pract [Internet]. 1996 [cited 2019 jun 7];10(3):257-81. Available from: https://www.ncbi.nlm.nih.gov/ pubmed/9009821

2. Fitzpatrick JJ, McCarthy G, editors. Nursing concept analysis: applications to research and practice. New York: Springer; 2016.

3. Meleis Al. Theoretical nursing: development and progress. 5th ed. Philadelphia: Lippincott; 2012.

4. Weaver K, Mitcham C. Nursing concept analysis in North America: State of the art. Nurs Philos. 2008;9(3):180-94. http://dx.doi.org/10.1111/j.1466769X.2008.00359.x. PMid:18582294.

5. Morse JM. Exploring pragmatic utility: concept analysis by critically appraising the literature. In: Rodgers B, Knafl KA. editors. Concept development in nursing: foundations, techniques and applications. 2nd ed. Philadelphia: Saunders; 2000. Chap. 17.

6. Penrod J, Hupcey JE. Enhancing methodological clarity: principlebased concept analysis. J Adv Nurs. 2005;50(4):403-9. http://dx.doi. org/10.1111/j.1365-2648.2005.03405.x. PMid:15842447.

7. Fawcett J, DeSanto-Madeya S. Contemporary nursing knowledge: analysis and evaluation of nursing models and theories. 3th ed. Philadelphia: SA Davis; 2013.

8. Rodgers BL. Concept analysis and the development of nursing knowledge: The evolutionary cicle. J Adv Nurs. 1989;14(4):330-5. http://dx.doi. org/10.1111/j.1365-2648.1989.tb03420.x. PMid:2661622.

9. Walker LO, Avant KC. Strategies for theory construction in nursing Norwalk: Appleton; 1983.

10. Walker LO, Avant KC. Strategies for theory construction in nursing. 6th ed. New York: Pearson; 2019.

11. McKenna H. Nursing theories and models. London: Routledge; 2000.

12. Cutcliffe JR, McKenna HP. The essential concepts of nursing: building blocks for practice. Edimburgh: Elsevier; 2005.

13. Hupcey JE, Morse JM, Lenz ER, Tason MC. Wilsonian methods of concept analysis: a critique. Sch Inq Nurs Pract. 1996;10(3):185-210. PMid:9009818.
14. Penrod J, Hupcey JE. Concept advancement: extending science through concept-driven research. Res Theory Nurs Pract. 2005;19(3):231-41. http://dx.doi.org/10.1891/rtnp.2005.19.3.231. PMid:16144241.

15. Zagonel IPS. Análise de conceito: um exercício intelectual em enfermagem. Cogitare Enfer [Internet]. 1996 [cited 2019 set 2]. 1(1):10-14. Disponível em: https://revistas.ufpr.br/cogitare/article/view/44944

16. Fernandes MGM, Nóbrega MML, Garcia TR, Macêdo-Costa KNF Conceptual analysis: methodological considerations. Rev Bras Enferm 2011;64(6):1150-6. http://dx.doi.org/10.1590/S0034-71672011000600024. PMid:22664616.

17. Bousso RS, Poles K, Cruz DALM. Nursing concepts and theories. Rev Esc Enferm USP. 2014;48(1):141-5. http://dx.doi.org/10.1590/S0080623420140000100018. PMid:24676120.

18. Brandão MAG, Mercês CAM, Lopes ROP, Martins JSA, Souza PA, Primo $\mathrm{CC}$. Concept analysis strategies for the development of middle-range nursing theories. Texto Contexto Enferm. 2019;28:e20180390. http:// dx.doi.org/10.1590/1980-265x-tce-2018-0390.

19. Knafl KA, Deatrick JA. Commentary on: Draper P. Editorial: a critique of concept analysis. J Adv Nurs. 2014;70(12):2968. http://dx.doi. org/10.1111/jan.12465. PMid:25378184.

20. Draper P. A critique of concept analysis. J Adv Nurs. 2014;70(6):1207-8 http://dx.doi.org/10.1111/jan.12280. PMid:24467340.

21. Bergdahl E, Berterö CM. Concept analysis and the building blocks of theory: misconceptions regarding theory development. J Adv Nurs. 2016;72(10):2558-66. http://dx.doi.org/10.1111/jan.13002. PMid:27218439.

22. Rodgers BL, Jacelon CS, Knafl KA. Concept analysis and the advance of nursing knowledge: state of the science. J Nurs Scholarsh. 2018;50(4):4519. http://dx.doi.org/10.1111/jnu.12386. PMid:29689127.

23. Schwartz-Barcott D, Kim HS. An expansion and elaboration of the Hybrid Model of Concept Development. In: Rodgers B, Knafl KA. (ed.). Concept development in nursing: foundations, techniques and applications. 2. ed. Philadelphia: Saunders; 2000. Chap. 9.

24. Rodgers BL. Concept analysis: An evolutionary view. In: Rodgers B, Knaf $\mathrm{KA}$, editors. Concept development in nursing: foundations, techniques and applications. 2nd ed. Philadelphia: Saunders; 2000. Chap. 6.

25. Wills EM, McEwen M. Concept development: clarify meanings of terms. In: McEwen M, Wills EM. Theoretical basis for nursing. 4th ed. Philadelphia: Lippincott Williams \& Wilkins; 2014. Chap. 3

26. Nuopponen A. Methods of concept analysis - A comparative study Part 1 of 3. LSP Journal [Internet]. 2010 [cited 2019 jun 4]; 1(1):4-12. Available from: https://rauli.cbs.dk/index.php/lspcog/article/view/2970

27. Nuopponen A. Methods of concept analysis - Towards systematic concept analysis (part 2 of 3). LSP Journal [Internet]. 2010 [cited 2019 jun 4]; 1(2):5-14. Available from: https://rauli.cbs.dk/index.php/lspcog/ article/view/3092

28. Nuopponen A. Methods of concept analysis - tools for systematic concept analysis (part 3 of 3). LSP Journal. 2011; 2(1):4-15. Available from: https://rauli.cbs.dk/index.php/lspcog/article/view/3302

29. Wilson J. Pensar com conceitos. 2. ed. São Paulo: Martins Fontes; 2005.

30. Morse JM. Exploring the theoretical basis of nursing using advanced techniques of concept analysis. ANS - Adv nurs sci. 1995; 17(3):31-46. http://dx.doi.org/10.1097/00012272-199503000-00005. 